\title{
A "EDIÇÃO JESUÍTA" DOS PRINCIPIA DE ISAAC NEWTON: UM TRABALHO DE RELEITURA
}

DOI: http://dx.doi.org/10.15448/2178-3748.2015.2.22226

\author{
Gustavo Rodrigues Rocha \\ Doutor - Universidade Estadual de Feira de Santana \\ E-Mail: gustavo.rodrigues.rocha@gmail.com
}

BUSSOTTI, Paolo; PISANO, Raffaele. Newton's Philosophiae Principia Mathematica "Jesuit" Edition: The Tenor of a Huge Work. Rendiconti Lincei Matematica e Applicazioni, Vol. 25, No 4, p. 413-444, 2014.

Os Principios Matemáticos da Filosofia Natural (em latim, Philosophiae Naturalis Principia Mathematica), doravante somente Principia, obra de três volumes escrita por Isaac Newton, e publicada em 1687, é a obra seminal da história da física moderna. O prêmio Nobel de Física, Steven Weinberg, teórico de campos, ao refletir sobre a Física, em seu livro de 1972, sobre cosmologia e gravitação, afirmou que "tudo o que aconteceu desde 1687 é um comentário (ou polimento) dos Principia" (apud PASK, 2013, p. 14). A segunda edição dos Principia foi publicada em 1713 - e reimpressa e corrigida em 1714 -, contendo uma teoria da Lua mais completa, assim como dos equinócios e dos cometas. A terceira edição dos Principia foi publicada em 1726. Newton acrescentou na terceira edição novas explicações sobre a resistência dos fluidos no Livro 2 (continuação do Livro 1, De motu corporum, em português, "Sobre o movimento dos corpos"), assim como explicações mais detalhadas sobre a órbita da Lua e o papel da gravitação, e, no Livro 3 (De mundi systemate, em português, "Sobre o sistema do Mundo"), novas observações sobre Júpiter e os cometas. A primeira tradução para o inglês foi publicada em 1729, por Andrew Motte, a partir da terceira edição dos Principia de 1726. A chamada "Edição Jesuíta", foco do projeto de Bussotti e Pisano, baseou-se também na terceira edição de Newton, publicada entre 1739 e 1742, em quatro volumes, pelos escolásticos Thomas Le Seur (1703-1770), François Jacquier (1711-1788), frades franceses, e Jean Louis Calandrini (1703-1758), matemático suíço. Esta edição contém um número de comentários, explicações e adendos aos Principia, já não mais do próprio punho de Newton. 
A obra de Newton tem sido desde então objeto de exegeses e estudos, traduções e comentários, interpretações e reformulações. Os procedimentos geométricos de Newton foram paulatinamente substituídos pelos procedimentos analíticos. Os editores da "Edição Jesuíta", por exemplo, utilizaram-se do formalismo de Leonhard Euler (1707-1783), delineado em sua obra Mechanica (1736), para reescrever em equações diferenciais as proposições dos Principia. Os trabalhos dos cientistas Pierre Varignon (1645-1722), David Gregory (16591708), Guillaume François Antoine de Sainte Mesme marquês de l'Hôpital (1661-1704), Johann Bernoulli (1667-1748), Abraham de Moivre (1667-1754), John Keil (1671-1748), Jacob Hermann (1678-1733) e Willem Jacob's Gravesande (1688-1742) estão entre as primeiras contribuições para a Física originadas da exegese e dos resultados expostos nos Principia. Além das mencionadas edições históricas, há várias edições modernas dos Principia. O trabalho de exegese da terceira edição de Newton pelos estudiosos, Bernard Cohen (1914-2003), conhecido pelas obras Introduction to Newton's Principia (1971) e The Newtonian Revolution (1980), e Alexandre Koyré (1892-1964), conhecido pela obra Newtonian Studies (1965) está entre um dos exemplos mais notáveis no século XX. O levantamento bibliográfico mais completo da literatura secundária sobre a vida e a obra de Newton até o período de Cohen e Koyré é Newton and Newtoniana 1672-1975, A Bibliography de Peter Wallis e Ruth Wallis.

$\mathrm{Na}$ historiografia newtoniana das últimas décadas, destacam-se os trabalhos de BLAY (1995), BRACKENRIDGE (1995), DE GANDT (1995), DENSMORE (1995) e DOBBS (1976, 1991, 1995). Os físicos Richard Dalitz e Michael Nauenberg - especialista em Hooke, Newton e Huygens - editaram, em 2000, o livro The Foundations of Newtonian Scholarship. O livro é fruto de um simpósio na Royal Society que buscou reunir os principais especialistas em estudos newtonianos da atualidade. A biografia de Newton de Richard Westfall (19241996), Never at Rest (1980), "baseada em um estudo extensivo dos manuscritos de Newton, não meramente delineia uma crônica dos eventos da vida e da obra de Newton, mas ilumina quase que todos os aspectos das suas realizações científicas" (BUCHWALD; COHEN, 2001, p. xiv). No campo das traduções e comentários, destaca-se a nova tradução dos Principia por Bernard Cohen e Anne Whitman de 1999. O estudo do físico indiano S. Chandrasekhar (1910-1995), Newton's Principia for the Common Reader (1995), destaca-se entre as releituras recentes dos Principia. Os trabalhos de divulgação científica dos Principia têm também uma longa tradição, desde o Newtonianism for Ladies (1737), do escritor italiano oitocentista Francesco Algarotti (1712-1764), até o recente Magnificent Principia: Exploring Isaac Newton's Masterpiece (2013), do matemático britânico Colin Pask (1943-). O trabalho 
do historiador da matemática Niccolò Guicciardi (1957-), autor de Reading the Principia (1999), tem também se tornado uma referência. A própria disciplina História da Ciência, como emerge a partir da segunda metade do século XX, também tem entre os seus clássicos exegeses dos Principia, como The Social and Economic Roots of Newton's Principia (1931), do historiador e filósofo da ciência Boris Hessen, que se tornou uma referência de estudos sociais das ciências.

É nesta tradição e neste esforço de exegese dos Principia que se insere o trabalho e o projeto de Paolo Bussotti, pesquisador e professor da Universidade de Udine, e Raffaele Pisano, pesquisador associado do Laboratório de História das Ciências e da Filosofia da Universidade de Lorraine. Thomas Le Seur e François Jacquier foram padres católicos da Ordem Minim - fundada no século XV na Itália - e erroneamente confundidos como jesuítas. A chamada "Edição Jesuíta", doravante somente EJ, é uma edição extensivamente comentada da terceira edição dos Principia, pouco estudada nesta tradição de exegese dos Principia. Os historiadores Bussotti e Pisano se colocaram a tarefa de preencherem esta lacuna. A EJ, depois de publicada, entre 1739 e 1742, teve várias reedições, como a edição de 1760 em Colônia, de 1780-85 em Praga, e as edições de 1822 e 1833 em Glasgow. Nesta terceira edição da EJ dos Principia, o matemático John Martin Frederick Wright, autor de $A$ Commentary on Newton's Principia (1833), corrigiu uma série de erros das edições anteriores. Esta terceira edição revisada da EJ dos Principia é o ponto de partida do trabalho de Bussotti e Pisano. A EJ continua sem tradução do original em latim. O projeto inclui uma tradução do latim para o inglês dos quatro volumes da EJ de 1822 adicionada de um quinto volume introdutório (projeto cuja previsão de conclusão é 2020).

Os dois autores, ao esclarecerem a importância da EJ, lembram aos seus leitores que, historicamente, os comentadores dos Principia buscaram "1) explicar as proposições de Newton de uma maneira mais clara que o próprio Newton; 2) traduzir as propriedades dadas por Newton geometricamente em termos mais analíticos; 3) explicar, eventualmente, o desenvolvimento das ciências físicas depois de Newton, baseadas nas descobertas de Newton" (BUSSOTTI; PISANO, 2014a, p. 35-36). Le Seur, Jacquier e Calandrini cumpriram esta tarefa de modo exemplar. Em primeiro lugar, os autores da EJ acrescentaram notas de esclarecimento para toda proposição de Newton nos Principia. Bussotti e Pisano lembram que não há nenhuma edição comentada dos Principia mais detalhada do que a EJ. Em segundo lugar, a partir da segunda metade do século XVIII, a obra supracitada de Euler, Mechanica (1736), e a EJ se tornaram as referências canônicas para os leitores dos Principia, uma vez que, cada vez mais, o formalismo de Newton, como expresso em sua magnum opus, tornava- 
se antiquado e obsoleto. Os trabalhos de Pierre Varignon (1654-1722), Jakob Bernoulli (16541705), David Gregory (1659-1708), Johann Bernoulli (1667-1748), John Keil (1671-1721), Jacob Hermann (1678-1744) e Daniel Bernoulli estão entre os primeiros que traduziram em termos completamente analíticos os resultados de Newton. Finalmente, os editores da EJ ofereceram um resumo enciclopédico dos resultados obtidos nas primeiras quatro décadas do século XVIII originados das aplicações dos cálculos de Newton nos Principia. O curioso é notar que a EJ permanece pouco diretamente comentada pela literatura secundária. Portanto, um trabalho de releitura da "Edição Jesuíta" dos Principia de Newton é de grande relevância para a historiografia newtoniana.

Bussotti e Pisano pretendem oferecer "três pedaços de informação fundamentais" a partir de seus estudos, a saber, "1) entender os resultados físicos e as técnicas matemáticas de Newton; 2) obter uma ideia clara do desenvolvimento da física e da análise matemática dentro dos 20 a 25 anos que se seguiram à publicação da terceira edição dos Principia; 3 ) compreender de forma mais abrangente a profunda diferença entre a abordagem físicamatemática de Newton e as abordagens dos seus sucessores" (BUSSOTTI; PISANO, 2014a, p. 37). Bussotti e Pisano propõem quatro linhas de pesquisa conectadas à EJ. A primeira linha de pesquisa está relacionada ao estudo das três personalidades da EJ. Por que os autores da EJ organizaram todo o aparato de notas da maneira como estão dispostas? A segunda linha de pesquisa está relacionada à política da ciência. Quem concebeu e quem financiou este empreendimento gigantesco? Quais são as razões por detrás desta escolha? Bussotti e Pisano pretendem reconstruir o ambiente social dentro do qual a EJ foi concebida, formulada e desenvolvida, assim como clarificar a natureza, o propósito e a estrutura das notas adicionadas pelos comentadores de Newton. A terceira linha de pesquisa se relaciona com as mudanças das ciências físicas e da matemática nas primeiras quatro décadas do século XVIII, o que levou à necessidade de um número crescente de comentadores a fim de tornar inteligível o estilo e a escrita newtoniana. Os dois historiadores pretendem verificar se há uma relação entre a mudança da matemática e o próprio conteúdo das teorias físicas neste período. A quarta linha de pesquisa, relacionada com a terceira, busca justamente saber qual era o público da EJ. "Quem deve ter sido os leitores da EJ?", perguntam os historiadores Bussotti e Pisano (BUSSOTTI; PISANO, 2014b, p. 439-440). Os dois autores prometem, portanto, uma série de artigos, nos anos vindouros, onde apresentarão seus resultados.

O artigo ora em questão, a saber, Newton's Philosophiae Principia Mathematica "Jesuit" Edition: The Tenor of a Huge Work, é dividido em duas partes. Na primeira parte, os dois historiadores dissertam sobre a estrutura geral da EJ, as personalidades dos três 
comentadores e o papel da EJ entre as edições e os comentários dos Principia entre o período de 1687 (que data a primeira edição dos Principia) e 1833 (que data a terceira edição corrigida da EJ). Na segunda parte, os dois autores delineiam o projeto editorial em andamento da edição traduzida e comentada da versão de Glasgow da EJ.

O primeiro volume da EJ foi publicado em 1739 e inclui o Livro 1 dos Principia. O segundo volume da EJ foi publicado em 1740 e inclui o Livro 2 dos Principia. O terceiro e o quarto volumes da EJ foram publicados em 1742. O terceiro volume inclui as 24 primeiras proposições do Livro 3 dos Principia. O quarto volume inclui as demais proposições e o General Scholium (um ensaio conclusivo que Newton inseriu a partir da segunda edição dos Principia). Há quatro tipos de comentários, sob o ponto de vista tipográfico, encontrados na "Edição Jesuíta". As notas que se referem diretamente a passagens de Newton e que são indicadas ao longo do texto de Newton por uma letra entre parênteses. As notas que não são interpretadas pelos comentadores como esclarecimentos diretos do texto de Newton. Os tratados que são inseridos pelos comentadores a fim de introduzirem problemas gerais tratados no texto de Newton e, finalmente, os tratados de outros autores que desenvolveram e aperfeiçoaram as teorias inicialmente delineadas pelo próprio Newton (o que se resume a três tratados sobre a teoria das marés, a saber, dos matemáticos Daniel Bernoulli, Colin McLaurin e Leonard Euler).

Na página título da EJ consta apenas os nomes dos frades franceses (que pode ser apreciada pela sua reprodução abaixo). No entanto, Le Seur e Jacuier reconhecem a contribuição de Calandrini ao final do primeiro livro. O tipógrafo da edição de Glasgow de 1822 é quem introduziu o erro de considerar jesuítas os editores franceses. Os dois são conhecidos basicamente pela EJ, porém também escreveram vários ensaios. Calandrini foi um newtoniano desde a juventude, tendo apresentado uma dissertação sobre as cores na Academia de Gênova. Calandrini se interessou, como matemático, pela trigonometria plana e esférica, pela teoria dos diferenciais e pelas séries infinitas. O matemático suíço também estudava botânica e meteorologia e se interessava pelos problemas da aurora boreal.

Bussotti e Pisano ilustram o potencial do trabalho que estão conduzindo ao apresentarem no artigo um exemplo que consideram paradigmático de comentário introduzido pelos editores da EJ. Este exemplo escolhido encontra-se no Livro 1, seção VII, proposição XLI, onde Newton trata da questão fundamental do problema do inverso das forças centrais. Bussotti e Pisano argumentam que este problema é paradigmático por representar a maneira característica da argumentação geométrica de Newton e a forma padrão como os comentadores trabalham para traduzi-la para a sua forma analítica moderna. Os dois 
historiadores enfatizam que este exemplo é importante para a história da matemática, pois representa uma transição de tradições de pensamento entre a época de Newton e aquela de seus sucessores imediatos. Depois de suas considerações finais, os autores deixam o leitor na expectativa, tanto pelos próximos resultados, quanto pelo projeto editorial final. Aguardemos atentos todos os desdobramentos desta importante contribuição para a historiografia newtoniana.
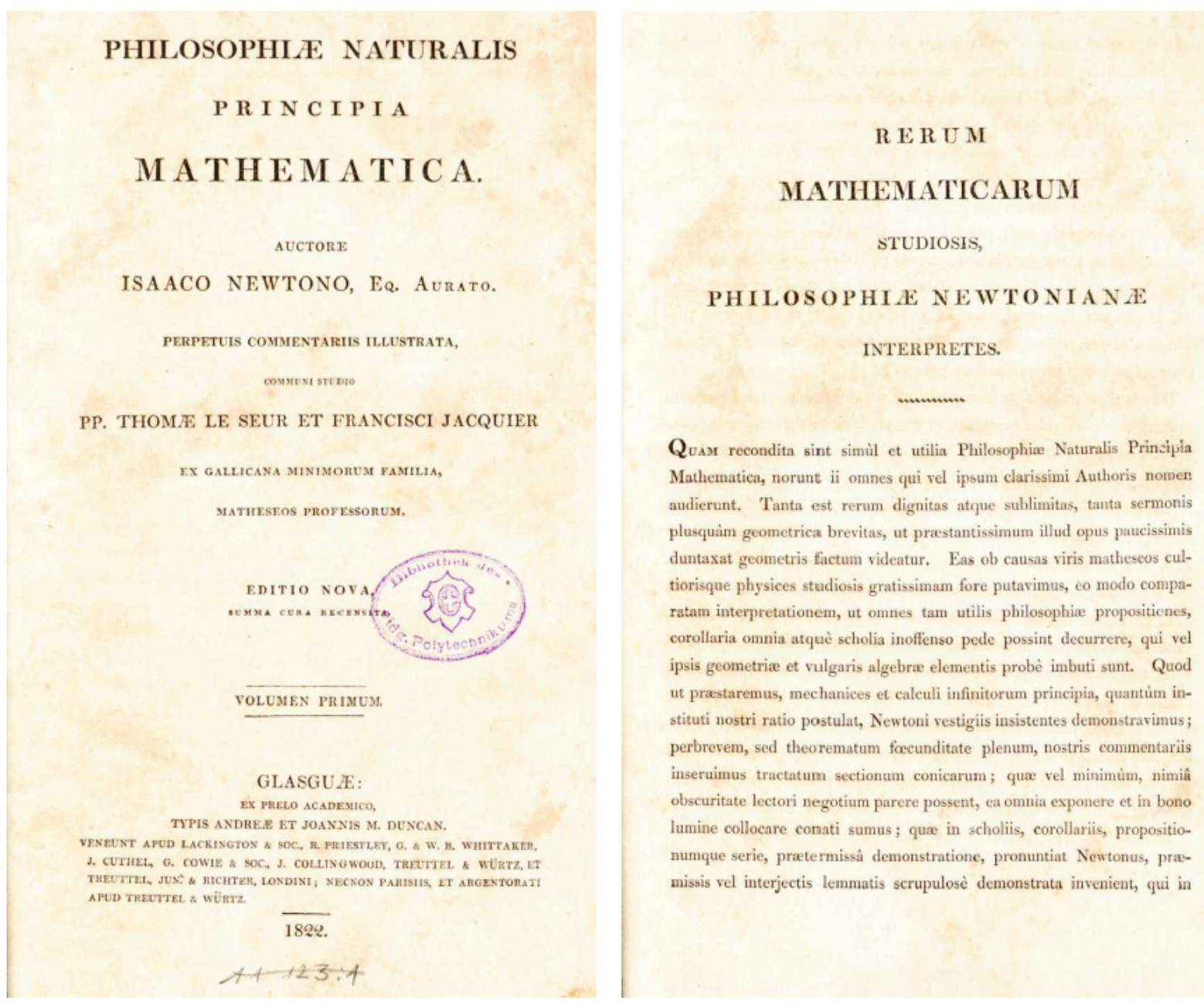

Página título da "Edição Jesuíta" de Glasgow de 1822. In: BUSSOTTI; PISANO, 2014, p. 36.

\section{Referências Bibliográficas}

BLAY, Michel. Les "Principia" de Newton. Paris: Presses Universitaires de France, 1995.

BRACKENRIDGE, J. Bruce. The Key to Newton's Dynamics: The Kepler Problem and the Principia. Berkeley: University of California Press, 1995.

BUCHWALD, Jed Z.; COHEN, I. Bernard (Org.). Isaac Newton's Natural Philosophy. Cambridge: The MIT Press, 2001. 
BUSSOTTI, Paolo; PISANO, Raffaele. Newton's Philosophiae Principia Mathematica "Jesuit" Edition: The Tenor of a Huge Work. Rendiconti Lincei Matematica e Applicazioni, Vol. 25, No 4, p. 413-444, 2014b.

BUSSOTTI, Paolo; PISANO, Raffaele. On the Jesuit Edition of Newton's Principia. Science and Advanced Researches in the Western Civilization. Advances in Historical Studies, Vol. 3, No1, p. 3355, 2014a.

CHANDRASEKHAR, S. Newton's Principia for the Common Reader. Oxford: Oxford University Press, 1995.

COHEN, I. Bernard. Introduction to Newton's Principia. Cambridge: Harvard University Press, 1971.

COHEN, I. Bernard. The Newtonian Revolution - with illustrations of the transformation of scientific ideas. Cambridge: Cambridge University Press, 1980.

COHEN, I. Bernard; WHITMAN, Anne (Org). Mathematical Principles of Natural Philosophy by Isaac Newton: A New Translation. Berkeley: University of California Press, 1999.

DALITZ, Richard; NAUENBERG, Michael (Org). The Foundations of Newtonian Scholarship. Singapura: World Scientific Publishing Co. Pte. Ltd, 2000.

DE GANDT, François. Force and Geometry in Newton's "Principia". New Jersey: Princeton University Press, 1995.

DENSMORE, Dana. Newton's Principia, The Central Argument: Translation, Notes, Expanded Proofs. Santa Fe: Green Lion Press, 1995.

DOBBS, Betty Jo Teeter. The Foundations of Newton's Alchemy. Cambridge: Cambridge University Press, 1976.

DOBBS, Betty Jo Teeter. The Janus Faces of Genius: The Role of Alchemy in Newton's Thought. Cambridge: Cambridge Unviersity Press, 1991.

DOBBS, Betty Jo Teeter; JACOB, Margaret C. Newton and the Culture of Newtonianism. New York: Prometheus Books, 1995.

GUICCIARDINI, Niccolò. Reading the Principia: The Debate on NEwton's MAthematical Methods for Natural Philosophy from 1687 to 1736. Cambridge: Cambridge University Press, 1999.

KOYRÉ, Alexandre. Newtonian Studies. Chicago: University Chicago Press, 1965.

PASK, Colin. Magnificent Principia: Exploring Isaac Newton's Masterpiece. Amherst: Prometheus Books, 2013.

WALLIS, Peter; WALLIS, Ruth. Newton and Newtoniana 1675-1975, A Bibliography. Folkestone: Dawson, 1977.

WESTFALL, Richard S. Never at Rest: A Biography of Isaac Newton. Cambridge: Cambridge University Press, 1980.

RESENHA ENVIADA EM: 22/10/2015

ACEITA PARA PUBLICAÇÃO EM: 19/11/2015 